\title{
Buffering plasmons in nanoparticle waveguides at the virtual-localized transition
}

\author{
Raúl A. Bustos-Marún, ${ }^{1,2}$ Eduardo A. Coronado, ${ }^{2}$ and Horacio M. Pastawski ${ }^{1}$ \\ ${ }^{1}$ IFEG and FAMAF, UNC, Ciudad Universitaria, 5000 Córdoba, Argentina \\ ${ }^{2}$ INFIQC, Departamento de Fisicoquímica, Facultad Ciencias Químicas, UNC, Ciudad Universitaria, 5000 Córdoba, Argentina
}

(Received 29 April 2010; published 26 July 2010)

\begin{abstract}
We study the plasmonic energy transfer from a locally excited nanoparticle (LE-NP) to a linear array of small NPs and we obtain the parametric dependence of the response function. An analytical expression allows us to distinguish the extended resonant states and the localized ones, as well as an elusive regime of virtual states. This last appears when the resonance width collapses and before it becomes a localized state. Contrary to common wisdom, the highest excitation transfer does not occur when the system has a well defined extended resonant state but just at the virtual-localized transition, where the main plasmonic modes have eigenfrequencies at the passband edge. The slow group velocity at this critical frequency enables the excitation buffering and hence favors a strong signal inside the chain. A similar situation should appear in many other physical systems. The extreme sensitivity of this transition to the waveguide and LE-NP parameters provides new tools for plasmonics.
\end{abstract}

DOI: 10.1103/PhysRevB.82.035434

PACS number(s): 73.21.-b, 42.79.Gn, 63.20.Pw, 78.67.-n

\section{INTRODUCTION}

Electromagnetic energy can be focused and guided below the light diffraction limit by transforming it into a collective plasmonic excitation, the surface plasmon polariton, that can propagate along a one dimensional array of nanoparticles (NPs). ${ }^{1}$ This feature has attracted significant attention due to its potential applications in optoelectronic devices, subwavelength waveguides, random lasers, optical traps, and hot-spot based plasmonic sensors for ultrasensitive spectroscopy. ${ }^{1-3}$ Previous works have already addressed the question of how to achieve a high degree of localization of plasmonic excitations ${ }^{4}$ and have studied the plasmon propagation on the waveguide formed by an ordered NP chain. ${ }^{2,5}$ However, a fundamental question remains open: how to transform a $l o-$ calized excitation into a strong signal somewhere else inside a finite chain. A natural idea, would be to exploit the divergent local density of states of one-dimensional (1D) systems, i.e., the vanishing group velocity at the band edge, as proposed for light buffering in photonic waveguides. ${ }^{6}$ However, such divergences are a bulk property absent in finite and semi-infinite chains, where the local density of states near the extremes cancels out at the passband edge. In a quantum tight-binding model, this corresponds to a semielliptical density of states. Therefore, the only alternative would seem to tailor the surface inhomogeneity to generate ad hoc resonances $^{7}$ that could easily be excited and transfer energy. This poses a big challenge for both experiments and numerical simulations, since there is a broad range of parameters to be explored. Thus, this work resorts to a model that, containing only essentials, could be solved analytically by using a response function formalism. Besides the expected resonant and localized eigenmodes, we find an elusive regime of virtual states. This appears when the resonance width collapses and before becoming a localized state, providing for a continuity between them. Quite surprisingly, we prove that virtual to localized states transition provides the route to optimal excitation transfer by recovering, at the chain extremes, a divergent local density of states with slow group velocity.
The work is organized as follows: In Sections II and III we present the model used to describe the system and develop a response function formalism and a pole analysis for it. Then, in Sec. IV we show and discuss the results, and finally, in Sec. V we summarize the main conclusions of the work.

\section{LINEAR RESPONSE IN NANOPARTICLE WAVEGUIDES}

The system studied, see Fig. 1, is a linear array of $N$ metal NPs coupled to a locally excited (LE) NP, described in the coupled dipole approximation. ${ }^{2,5,8,9}$ The induced dipole moment $\vec{P}_{i}$ at $i^{\text {th }}$ NP satisfies:

$$
\begin{aligned}
& {\left[\omega_{\mathrm{SP} i}^{2}-\omega^{2}-\mathrm{i} \eta_{i} \omega\right] \vec{P}_{i}} \\
& \quad=\frac{1}{3} r_{i}^{3} \omega_{\mathrm{P} i}^{2} 4 \pi \epsilon_{0}\left[\vec{E}_{i}^{(\mathrm{ext})}+\sum_{j \neq i}^{N} \vec{E}_{j, i}\left(\vec{P}_{j}, \vec{d}_{j, i}, \vec{k}\right)\right] .
\end{aligned}
$$

Here, $r_{i}, \omega_{\mathrm{P} i}, \omega_{\mathrm{SP} i}$, and $\eta_{i}$ correspond to the radius, bulk and surface plasmon frequencies, and electronic damping. $\epsilon_{0}$ is the free space permittivity. $\vec{E}_{i}^{(\text {ext })}$ and $\vec{E}_{j, i}$ are, respectively, the external field and the electric field at the $i$ th site produced by the $j$ th NP. In general, $\vec{E}_{j, i}$ is a complex function that depends on the separation vector $\vec{d}_{j, i}=d_{j, i} \hat{d}_{j, i}$ between NPs and the wave vector $\vec{k}$. However, if $d$ is small, $\vec{E}_{j, i}$ can be evaluated in the near field approximation,

$$
\vec{E}_{j, i}\left(\vec{P}_{j}, \vec{d}_{j, i}, \vec{k}\right) \stackrel{k d \rightarrow 0}{\approx} \frac{\vec{P}_{j}-3 \hat{d}_{j, i}\left(\vec{P}_{j} \cdot \hat{d}_{j, i}\right)}{4 \pi \epsilon_{0} n^{2} d_{j, i}^{3}},
$$

where $n$ is the refractive index of the host material. For a linear array of NPs, plasmon oscillations can only be transverse $(T)$ or longitudinal $(L)$ to the chain axis, and due to the cubic dependence of $E$ on $d$, it is a good approximation to neglect contributions beyond nearest neighbors. ${ }^{2}$ Arranging all $\vec{P}_{i}$ and $\vec{E}_{i}^{(\text {ext) }}$ as vectors $\mathbf{P}$ and E, Eq. (1) reads: 


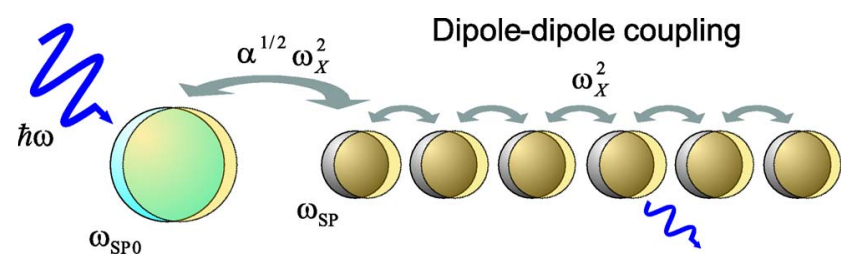

FIG. 1. (Color online) An external source $(\hbar \omega)$ excites the surface plasmon $\left(\omega_{\mathrm{SP} 0}\right)$ of a NP which is coupled, through $\alpha^{1 / 2} \omega_{\mathrm{X}}^{2}$, to a NP waveguide, of bandwidth $\left|\omega^{2}-\omega_{\mathrm{SP}}^{2}\right| \leq 2 \omega_{\mathrm{X}}^{2}$, where detection takes place.

$$
\mathbf{P}=\left(\llbracket \omega^{2}-\mathbb{M}\right)^{-1} \mathbb{R} \mathbf{E} \equiv \chi \mathbf{E},
$$

where $\mathrm{R}$ is a diagonal matrix with $R_{i, i}=-(4 / 3) \pi r_{i}^{3} \omega_{\mathrm{P} i}^{2} \epsilon_{0}$ and $\mathrm{M}$ is a tridiagonal matrix, with

$$
M_{0,0} \equiv \widetilde{\omega}_{\mathrm{SP} 0}^{2}=\omega_{\mathrm{SP} 0}^{2}-\mathrm{i} \eta_{0} \omega
$$

for the LE-NP, while for any of the equidistant identical NPs along the chain

$$
M_{i, i} \equiv \widetilde{\omega}_{\mathrm{SP}}^{2}=\omega_{\mathrm{SP}}^{2}-\mathrm{i} \eta \omega, \quad(\forall i \neq 0) .
$$

The dipole-dipole coupling strengths are

$$
M_{i, j} \equiv \omega_{\mathrm{X} i, j}^{2}=\frac{\gamma^{T, L} \omega_{\mathrm{P} i}^{2}}{3 n^{2}}\left(\frac{r_{i}}{d_{i, j}}\right)^{3},
$$

with $\gamma^{T}=1$, and $\gamma^{L}=-2$. Since we are interested in the particular case where only the LE-NP is different from the rest, it is convenient adopt the nearest-neighbor coupling as $\omega_{\mathrm{X} i, j}^{2}=\omega_{\mathrm{X}}^{2}$ for $i$ and $j \neq 0$.

This description is accurate for: (1) $k d \ll 1$ and (2) $r / d \lesssim 1 / 3$, where higher order multipoles are negligible. ${ }^{10}$ These conditions require small $r$ 's and hence a negligible radiation damping correction. ${ }^{8}$

Clearly, $\chi$ is a response function (RF), hence excitation dynamics between the different sites $i$ and $j$ is determined by the corresponding matrix elements of $\chi$. In this way, the square dipole moment of the $m^{\text {th }} \mathrm{NP},\left|P_{m}\right|^{2}$, produced when the LE-NP is externally excited with an electric field $E_{0}$, is

$$
\left|P_{m}\right|^{2}=\left|\chi_{m 0}\right|^{2}\left|E_{0}\right|^{2} \text {. }
$$

Notice that $\left(\mathbb{\omega} \omega^{2}-\mathbb{M}\right)^{-1}$ can be identified with a Green's function. ${ }^{11,12}$ The precise correspondence is presented in Table I and serves to exploit the analytical tools developed in this context. Compare this with the equivalence between electrons in a crystal and an array of coupled pendula as described in Sec. 5.2 of Ref. 11 and other textbooks.

\section{POLE ANALYSIS OF THE RESPONSE FUNCTION}

Since $\mathrm{M}$ is tridiagonal, $\chi_{i j}\left(\omega^{2}\right)$ admits exact analytical expressions as continued fractions. ${ }^{12,13}$ For finite systems, $\chi_{i j}\left(\omega^{2}\right)$ has a set of isolated poles at the eigenvalues of $\mathbb{M}$ whose real and imaginary parts are, respectively, the eigenfrequencies and their damping. Extending $\mathbb{M}$ to an infinite case enabled us to find an explicit expression for the response of our system at an arbitrary position $m$ given an excitation at position $i=0$,

$$
\chi_{m 0}(\omega)=\chi_{00}(\omega) \alpha_{1,0}^{1 / 2} e^{-m / \xi(\omega)},
$$

where

$$
\alpha_{1,0}=\omega_{\mathrm{X} 1,0}^{4} / \omega_{\mathrm{X}}^{4},
$$

accounts for the surface asymmetry, while

$$
\xi^{-1}(\omega)=\ln \left(\omega_{\mathrm{X}}^{2} / \Pi\right)=\kappa \pm i k
$$

is a generalized wave vector. The term $\chi_{00}$ is the RF of the LE-NP,

$$
\chi_{00}=\frac{R_{00}}{\left[\omega^{2}-\widetilde{\omega}_{\mathrm{SP} 0}^{2}\right]-\alpha \Pi(\omega)} .
$$

where the factor

$$
\alpha=\sqrt{\alpha_{1,0} \alpha_{0,1}}
$$

accounts for the effective coupling strengths, i.e., $\alpha=0$ describes an isolated LE-NP. For small $\alpha$, the peak at $\widetilde{\omega}_{\mathrm{SP} 0}^{2}$ is further shifted and broadened by $\alpha \Pi(\omega)$, a complex "selfenergy" accounting for the linear array. When $N$, the number of NPs, goes to infinite:

$$
\Pi(\omega)=\frac{1}{2}\left[\omega^{2}-\widetilde{\omega}_{\mathrm{SP}}^{2}\right]-\operatorname{sgn}\left(\omega^{2}-\omega_{\mathrm{SP}}^{2}\right) \frac{1}{2} \sqrt{\left[\omega^{2}-\widetilde{\omega}_{\mathrm{SP}}^{2}\right]^{2}-4 \omega_{\mathrm{X}}^{4}}
$$

In the weak damping limit (WDL), i.e., $\eta \rightarrow 0^{+}$, the propagating frequencies are given by the usual dispersion relation, ${ }^{2}$

$$
\omega^{2}(k)=\omega_{\mathrm{SP}}^{2}-2 \omega_{\mathrm{X}}^{2} \cos (k d),
$$

with wave number $k \in[-\pi / d, \pi / d]$. Within the "passband" $\left|\omega^{2}-\omega_{\text {SP }}^{2}\right| \leq 2 \omega_{X}^{2}$, each frequency component of the excitation propagates with group velocity

$$
v_{g}=\frac{d}{2 \omega} \sqrt{4 \omega_{\mathrm{X}}^{4}-\left[\omega^{2}-\omega_{\mathrm{SP}}^{2}\right]^{2}} .
$$

Components outside the passband decay exponentially along the chain within the localization length $\kappa^{-1}$. The inclusion of electronic damping $\eta$ adds a further decay and smears out the dispersion relation. The overall behavior of Eq. (8) is consistent with the numerical solutions including full retardation effects under similar conditions. ${ }^{9}$

The local density of plasmonic states (LDPS) at site $i=0$ is given by $\operatorname{Im}\left(\chi_{00}\right)$. In the WDL, LDPS quantifies the participation of this site on the different eigenfrequencies in a range $\mathrm{d} \omega$ around $\omega$. More generally, for finite $\eta, \omega \operatorname{Im}\left(\chi_{00}\right)$ is proportional to the power absorbed when site 0 is irradiated with frequency $\omega$.

The case $\alpha=0$ of Eq. (11) exemplifies the general behavior of finite systems, where poles of the RP (zeros in the denominator of $\chi_{00}$ ) determine the frequencies of maximum energy absorption. In this situation, dissipation occurs due to the damping processes accounted by $\eta$. In an infinite system, a new mechanism appears as $\operatorname{Im}(\Pi)$ also describes the irreversible energy spread through the chain. In this case, Eq. (11) has "poles," $\omega_{\text {pole }}$, which solve: 


$$
\begin{gathered}
{\left[\omega_{\text {pole }}^{2}-\omega_{\mathrm{SP} 0}^{2}-\frac{\alpha}{2}\left(\omega_{\text {pole }}^{2}-\omega_{\mathrm{SP}}^{2}\right)\right]^{2}} \\
=\frac{\alpha^{2}}{4}\left[\left(\omega_{\text {pole }}^{2}-\omega_{\mathrm{SP}}^{2}\right)^{2}-4 \omega_{\mathrm{X}}^{4}\right] .
\end{gathered}
$$

The solution of this equation is:

$$
\widetilde{\omega}_{\text {pole }}^{2}=\frac{[\beta-\alpha(\beta+1) / 2]}{(1-\alpha)} \pm \frac{\alpha}{2(1-\alpha)} \sqrt{(1-\beta)^{2}-4 V^{2}(1-\alpha)},
$$

where $\widetilde{\omega}_{\text {pole }}^{2}=\frac{\omega_{\text {pole }}^{2}}{\widetilde{\omega}_{\mathrm{SP}}^{2}}, \beta=\frac{\widetilde{\omega}_{\mathrm{SP} 0}^{2}}{\widetilde{\omega}_{\mathrm{SP}}^{2}}$, and $V=\frac{\omega_{\mathrm{X}}^{2}}{\widetilde{\omega}_{\mathrm{SP}}^{2}}$. The analysis of the different types of "poles" or solutions of Eq. (17) is simple in the WDL. In this case, two types of nonphysical poles appear: (1) The pole has a positive imaginary part. (2) The pole is real but it corresponds to a divergence of the original equation $\left(\chi_{00}\right)$ with a nonphysical self-energy $[\operatorname{Im}(\Pi)<0]$. This second alternative can appear because, in order to obtain a closed expression for $\omega_{\text {pole }}$, the denominator of $\chi_{00}$ should be squared [Eq. (16)]. This makes physical and nonphysical self-energies indistinguishable. Therefore, both solutions are present in Eq. (17). The second type of nonphysical poles are associated with virtual states, because even though they do not appear as resonances, they affect the LDPS within the passband, in the same way as localized or "real" states.

Figure 2 shows the real part of the poles of the response function, $\omega_{\text {pole }}$, as function of $\alpha^{1 / 2}$ for four different values of $\omega_{\mathrm{SP} 0}$. The figure also compares the case of the analytical solution resulting from the WDL of Eq. (17) with its numerical evaluation for a case with realistic damping. Numerical evaluation is required for $\eta \neq 0$ because $\beta, V$, and $\widetilde{\omega}_{\text {pole }}^{2}$ depend on $\omega$ in this case. Notice the accuracy of the WDL approximation. These poles provide for discrete localized levels (L), resonant levels (R), and virtual states (V) according to the parametric region.

In the WDL, when a pole is complex with a negative imaginary part, its real part lies within the passband and corresponds to the eigenfrequency of the LE-NP (Fig. 3), while its imaginary part roughly represents the decay rate. This is the case of a resonant state. When the pole is real, the usual situation is that the system has a localized eigenmode whose eigenfrequency lies outside the passband (Fig. 3). An excitation at this frequency will remain indefinitely within the localization length $1 / \kappa$. These two situations would typically exhaust the analysis. However, for quantum systems it has recently become clear that the transition between these two regimes, although covering a very narrow parametric range, has subtle and unique properties: there is a real "pole" which nevertheless does not correspond to an eigenstate of the system (see Refs. 14 and 15). As such, one might not know what to expect. This situation worsens in a plasmonic case where such parametric region broadens. Even though, they are not physical poles, in fact they do not solve $\left[\omega^{2}\right.$ $\left.-\widetilde{\omega}_{\mathrm{SP} 0}^{2}\right]-\alpha \Pi=0$, they still affect the LDPS, and hence the RF, within the passband (Fig. 3) in a similar way as localized states. In both cases, the LDPS is modulated at the band edges by a divergent factor $1 /\left(\omega^{2}-\omega_{\text {pole }}^{2}\right)$.
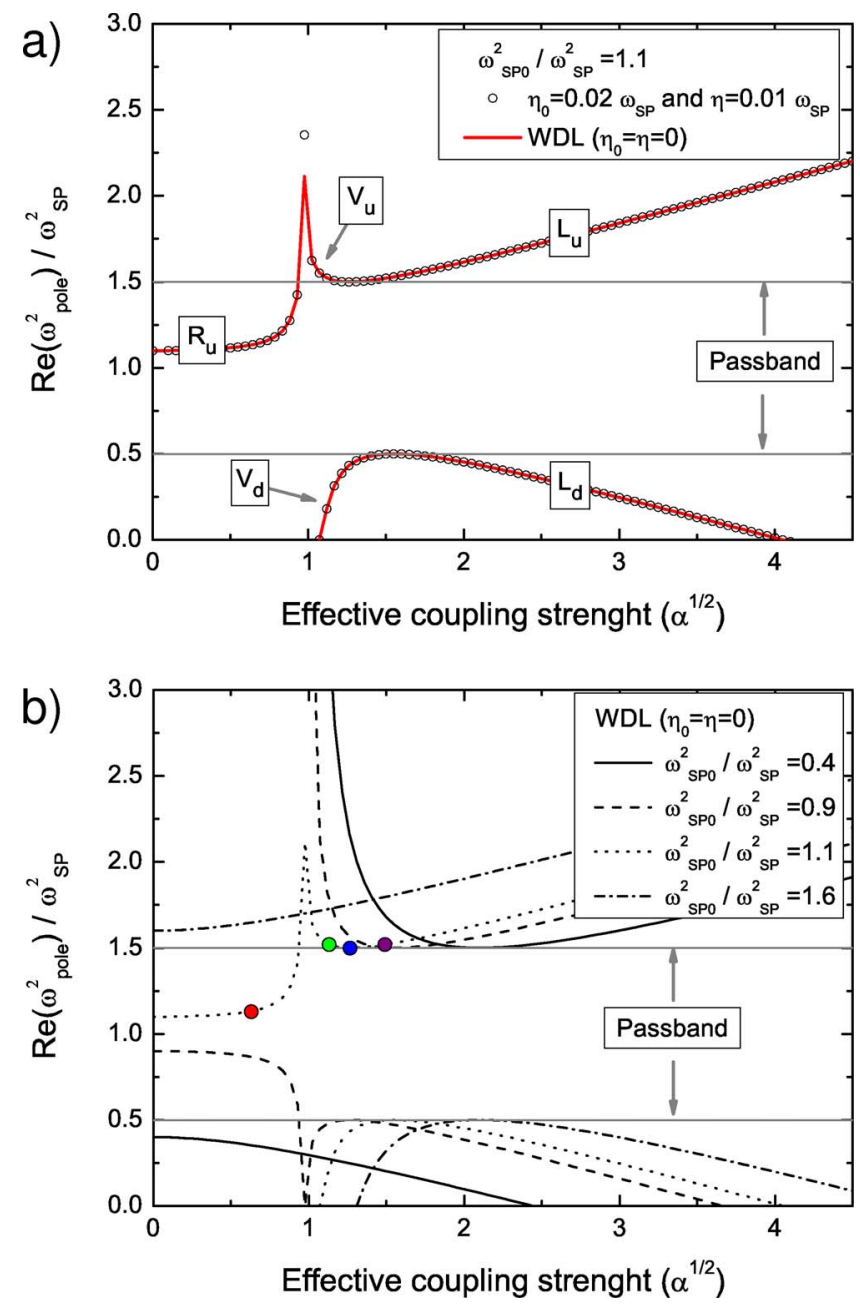

FIG. 2. (Color online) Real part of poles of $\chi$ as function of $\alpha^{1 / 2}$. Here, $\omega_{\mathrm{X}}^{2} / \omega_{\mathrm{SP}}^{2}=0.25$. In (a) three different regimes are shown: discrete localized levels $\left(\mathrm{L}_{d}\right.$ and $\left.\mathrm{L}_{u}\right)$, resonant level $\left(\mathrm{R}_{u}\right)$ and virtual states $\left(\mathrm{V}_{d}\right.$ and $\left.\mathrm{V}_{u}\right)$. Subscript $\mathbf{u}$ and $\mathbf{d}$ stand for "up" and "down," the possible positions of $\operatorname{Re}\left(\omega_{\text {pole }}^{2}\right)$ relative to $\omega_{\mathrm{SP}}^{2}$. The numerical solutions for finite $\eta$ are compared with the analytical WDL. In panel (b) we explore the dependence of the poles on $\omega_{\mathrm{SP} 0}$. The colored dots indicate the parameters used in the discussion of the LDPS (see Fig. 3).

This relationship between the poles of RF and the LDPS of the LE-NP, can be explicitly written in the WDL,

$$
\mathrm{LDPS}=\frac{R_{00}}{\omega_{\mathrm{SP}}^{2}} \times N(\omega) \times c \times L(\omega),
$$

where $c$ is a normalization constant

$$
c=\frac{2 V^{2}}{\sqrt{4 V^{2}(1-\alpha)-(1-\beta)^{2}}},
$$

$L$ is a Lorentzian function (in a plot as function of $\widetilde{\omega}^{2}$ $\left.=\omega^{2} / \omega_{\mathrm{SP}}^{2}\right)$, 


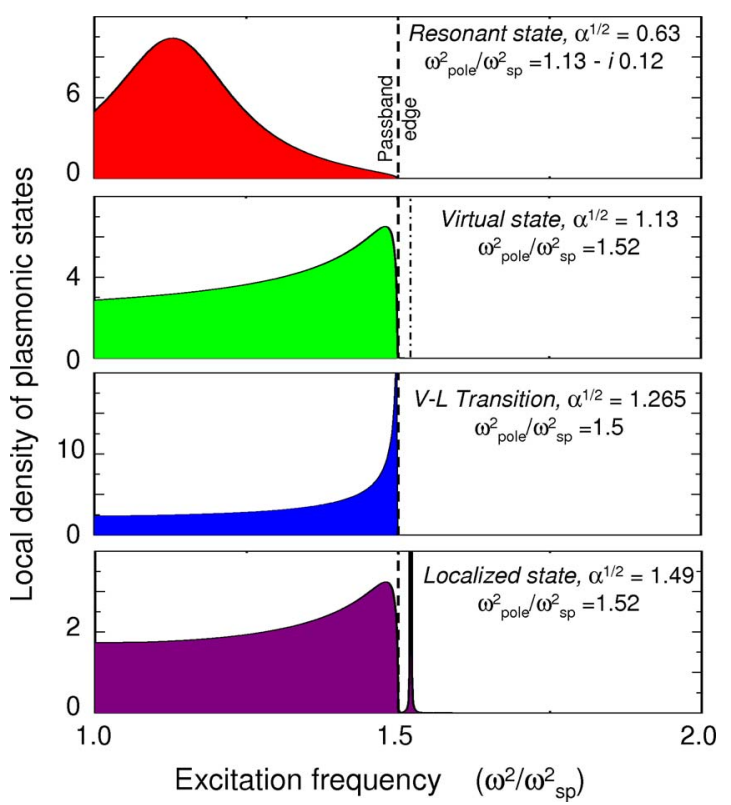

FIG. 3. (Color online) LDPS as function of the excitation frequency for different $\alpha$ values. Here, $\omega_{\mathrm{SP} 0}^{2} / \omega_{\mathrm{SP}}^{2}=1.1$ and $\omega_{\mathrm{X}}^{2} / \omega_{\mathrm{SP}}^{2}$ $=0.25$. Also, $\eta=\eta_{0}=0$ except for the panel showing a localized state case, where $\eta=10^{-5}$. Notice the identical shape of the LDPS near the passband edge for both virtual and localized states, and the divergence at the virtual-localized transition.

$$
L(\omega)=\frac{\widetilde{\Gamma}^{2}}{\left(\widetilde{\omega}^{2}-\widetilde{\omega}_{0}^{2}\right)^{2}+\widetilde{\Gamma}^{4}},
$$

and $N(\omega)$ is proportional to the LDPS at the surface site of a semi-infinite chain of identical NPs,

$$
N(\omega)=\frac{\sqrt{4 V^{2}-\left(1-\tilde{\omega}^{2}\right)^{2}}}{2 V^{2}} .
$$

In the resonant state regime, the maximum and the width of $L(\omega)$ are given by the real and imaginary part of $\widetilde{\omega}_{\text {pole }}^{2}$,

$$
\widetilde{\omega}_{0}^{2}=\left(\frac{\omega_{0}}{\omega_{\mathrm{SP}}}\right)^{2}=\frac{[\beta-\alpha(\beta+1) / 2]}{(1-\alpha)}
$$

and

$$
\tilde{\Gamma}^{2}=\left(\frac{\Gamma}{\omega_{\mathrm{SP}}}\right)^{2}=\frac{\alpha}{2(1-\alpha)} \sqrt{4 V^{2}(1-\alpha)-(1-\beta)^{2}} .
$$

In the virtual and localized state regimes, these expressions are still valid but $L(\omega)$ is no longer a Lorentzian function as $\widetilde{\Gamma}^{2}$ is imaginary. In this case, it is better to write the LDPS at the LE-NP as

$$
\operatorname{LDPS}=\frac{R_{00}}{\omega_{\mathrm{SP}}^{2}} \times N(\omega) \times \frac{\alpha V^{2}}{(1-\alpha)} \frac{1}{\left(\widetilde{\omega}^{2}-\widetilde{\omega}_{\text {pole }+}^{2}\right)\left(\widetilde{\omega}^{2}-\widetilde{\omega}_{\text {pole }-}^{2}\right)} .
$$

Notice the divergences that appear at the virtual-localized transition, i.e., when $\omega_{\text {pole }}$ reaches the passband edge. These divergences will strongly favor the excitation transfer. Figure 4 illustrates the frequency dependent excitation transfer
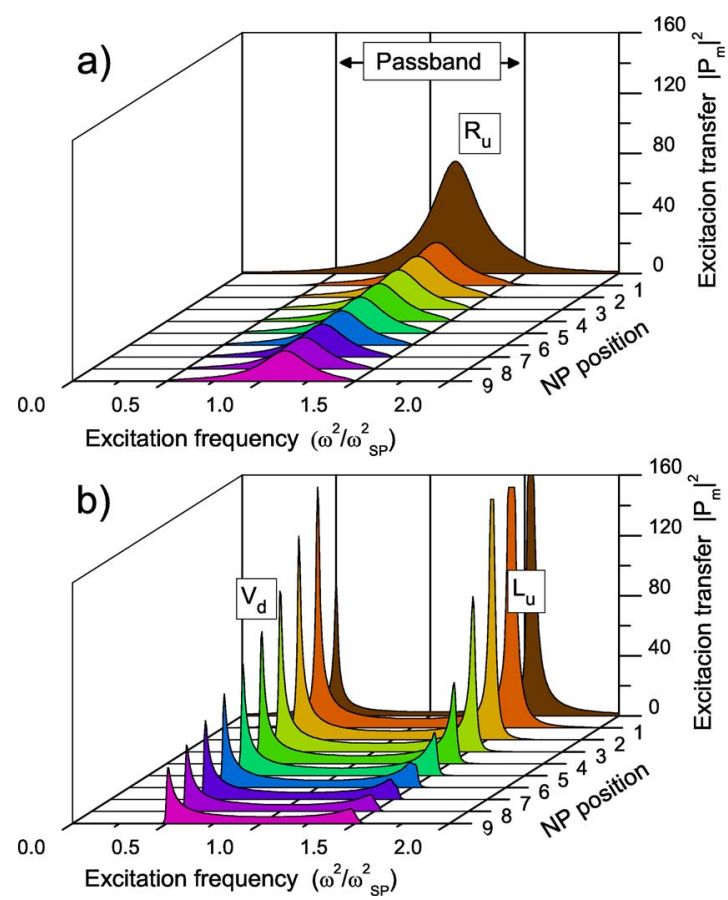

FIG. 4. (Color online) Square dipolar moment (in arbitrary units) as function of the NP position and excitation frequency. Here, $\eta=0.01 \omega_{\mathrm{SP}}, \quad \eta_{0}=0.02 \omega_{\mathrm{SP}}, \omega_{\mathrm{SP} 0}^{2} / \omega_{\mathrm{SP}}^{2}=1.1$, and $\omega_{\mathrm{X}}^{2} / \omega_{\mathrm{SP}}^{2}=0.25$. In subpanel (a) $\alpha^{1 / 2}=0.63$ and in subpanel (b) $\alpha^{1 / 2}=1.55$. L, V , and $\mathbf{R}$ stand for localized, virtual and resonant states, respectively, while $\mathbf{u}$ and $\mathbf{d}$ stand for up and down, the position of the pole relative to $\omega_{\mathrm{SP}}$.

along the chain for two cases: (a) a system with a resonant state and (b) a system with both, a virtual and a localized state.

As occurs for the oscillating-overdamped transition, and many of the spectral bifurcations omnipresent in nonHermitian Hamiltonians, ${ }^{16}$ the critical parameters of Eq. (17) occur when the argument under a square root vanishes and they identify the phase transition in the dynamical

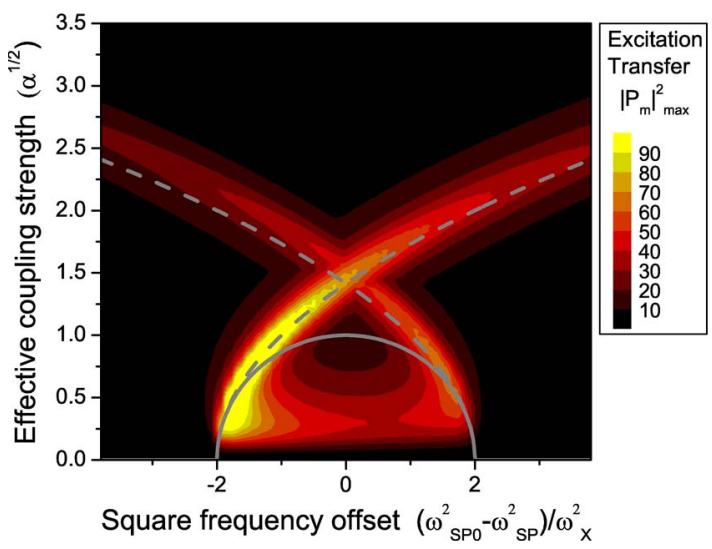

FIG. 5. (Color online) Color scale shows the maximum excitation transfer to the $5^{\text {th }} \mathrm{NP}\left(\max \left|P_{5}(\omega)\right|^{2}\right.$ in arbitrary units) for $\eta$ $=0.01 \omega_{\mathrm{SP}}$ and $\eta_{0}=0.02 \omega_{\mathrm{SP}}$. Continuous line delimits the region of resonant states [Eq. (25)] and dash lines mark the virtual-localized transitions [Eq. (26)], both evaluated in the WDL. 
TABLE I. Equivalence between plasmonic and quantum mechanical magnitudes.

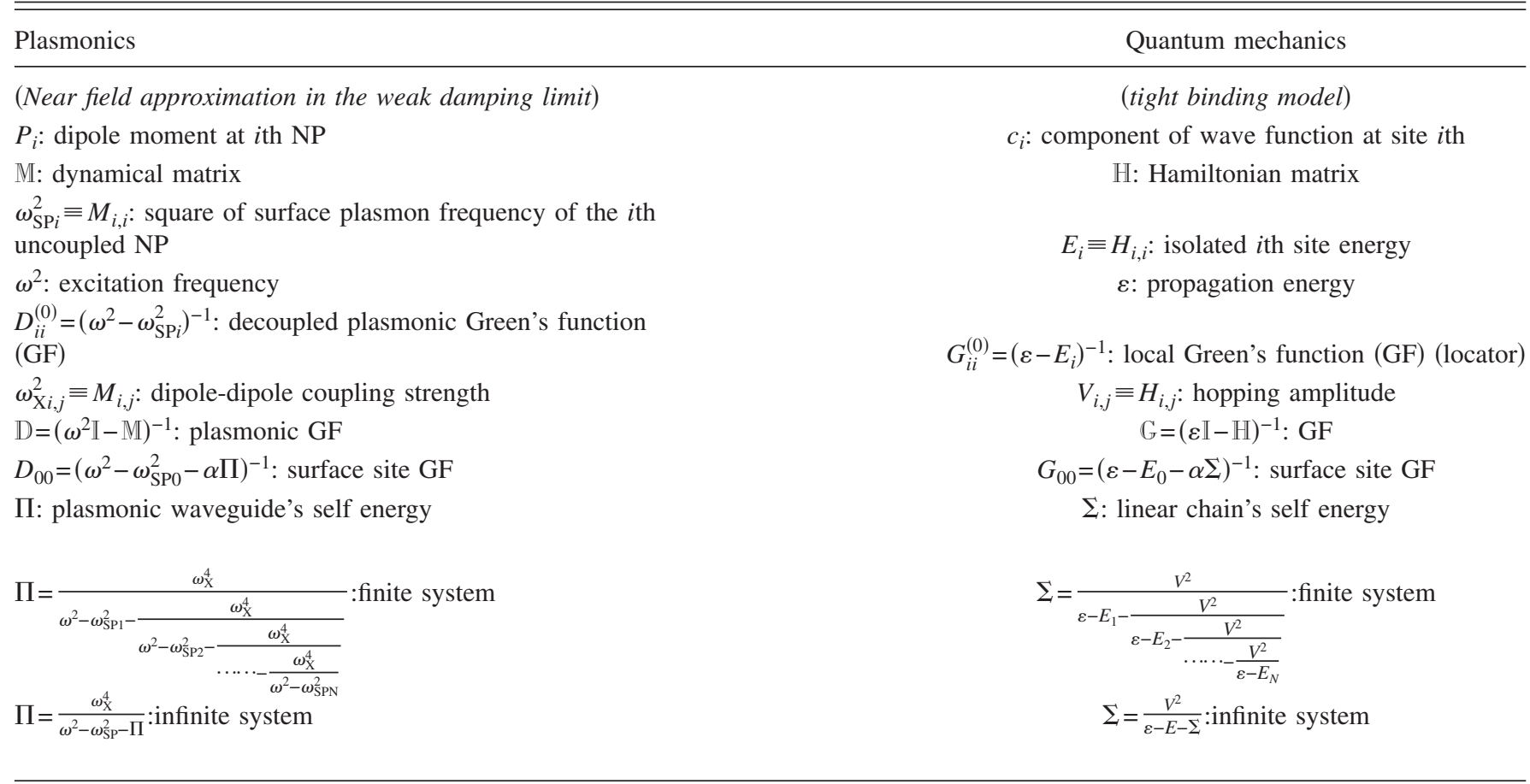

behavior. $^{17}$ The transition resonant-virtual occurs for $\operatorname{Im}\left(\omega_{\text {pole }}\right)=0$ :

$$
\alpha_{c 1}=\frac{2 \beta+4 V^{2}-\beta^{2}-1}{4 V^{2}},
$$

and the transition virtual-localized takes place when $\omega_{\text {pole }}$ $\in \mathfrak{R}$ and $\widetilde{\omega}_{\text {pole }}^{2}=1 \pm 2 \mathrm{~V}$,

$$
\alpha_{c 2( \pm)}=2 \pm \frac{(1-\beta)}{V} .
$$

These expressions, obtained in the WDL, are helpful to assess the role of the poles in the excitation transfer.

\section{OPTIMAL EXCITATION TRANSFER}

Figure 5 shows the maximum excitation transfer, $\left|P_{m}\right|^{2}$, enabled by a variation of $\omega$ at each system configuration. Superposed are the critical values resulting from Eqs. (25) and (26). Consistently with the above discussion, an appreciable transfer occurs in the resonant state regime. However, the maximum appears at the transition between virtual and localized states. Note that the optimal configuration for excitation transfer does not occur for $\omega_{\mathrm{SP} 0}=\omega_{\mathrm{SP}}$ and $\alpha=1$, where the LE-NP is indistinguishable from the others, as one might naïvely expect. Instead, it occurs for the highly asymmetric configuration where a virtual-localized transition appears. In this case, an excitation of a local mode that is coupled to collective excitations at the band edge, has components with very slow group velocity. In consequence, under a continuous irradiation, the excitation can build up. This dynamical interpretation is consistent with the new strategy developed in the context of photonic crystals. There, waveguides with slow group velocities are used as a way of buffering light. ${ }^{6}$ Alternatively, one sees that just at the virtual-localized transition, the spectroscopic Eq. (8) favorably combines its three factors: the response function on the excited nanoparticle $\chi_{00}(\omega)$ has a high intensity peak, this peak is inside the passband, and the relative effective coupling $\alpha_{1,0}^{1 / 2}$ is strong. Notice that a high transfer efficiency could be achieved even for $\omega_{\mathrm{SP} 0}$ so different from $\omega_{\mathrm{SP}}$ that the system could never form a resonant state just by changing $\alpha$. In analogy with the adatom in an Anderson-Newns model, ${ }^{14}$ a strong interaction with the substrate captures a state from the continuum spectrum to build a second localized state that would constitute the "antibonding" orbital of a dimer. ${ }^{18}$ This occurs through a virtual-localized transition and thus leads to the optimal excitation transfer shown in Fig. 5. Experimentally, these critical points could be achieved by properly tuning the distance, radius, shape and material of the NPs. Additionally, this configuration acts as a very narrow filter for the external frequency in resonance with the passband edge [see Fig. 4(b)]. The control of this critical phenomenon opens up many possibilities for applications. For example, the extreme sensibility of excitation transfer on $d_{0,1}$ when the system is close to a critical transition, would enable a new form of plasmon ruler suitable for biological and chemical applications. ${ }^{19}$ This occurs because $\alpha$ varies with $d_{0,1}^{-6}$ and, depending on $\omega_{\mathrm{SP} 0}, \omega_{\mathrm{SP}}$, and $\omega_{\mathrm{X}}^{2}$ which give the frequency offset of Fig. 5, the system response sweeps through different regimes within a narrow interval of $\alpha$. Similarly, as small changes on the refractive index modify dramatically the coupling $\omega_{X}^{2}$ and hence the passband, the excitation transfer will also be extremely sensitive to the dielectric environment in a system tuned with the virtual-localized transition. 


\section{CONCLUSIONS}

We have demonstrated that, contrary to common wisdom, the highest excitation transfer does not occur for a system with a well defined resonance but when a virtual state is transformed into a localized collective plasmonic mode whose eigenfrequency is just at the passband edge. The slow group velocity of an excitation with this critical frequency enables the excitation buffering and hence favors a strong signal inside the chain. The extreme sensitivity of this transition to the waveguide and LE-NP parameters should pro- vide new tools for plasmonics. As the basic model is quite general, our conclusions are universal in nature and apply to any of the broad class of systems that can be mapped to a linear array of damped oscillators. ${ }^{20}$

\section{ACKNOWLEDGMENTS}

The authors acknowledge the financial support from CONICET, SeCyT-UNC, ANPCyT, and MinCyT-Córdoba. Discussions with Axel Dente and Hernán Calvo are greatly acknowledged.
${ }^{1}$ S. A. Maier, Plasmonics: Fundamentals and Applications (Springer Press, New York, 2007); L. Novotny and B. Hecht, Principles of Nano-Optics (Cambridge University Press, Cambridge, England, 2007).

${ }^{2}$ M. L. Brongersma, J. W. Hartman, and H. A. Atwater, Phys. Rev. B 62, R16356 (2000).

${ }^{3}$ L. Burin, H. Cao, G. C. Schatz, and M. A. Ratner, J. Opt. Soc. Am. B 21, 121 (2004); M. Guillon, Opt. Express 14, 3045 (2006); S. Zou and G. C. Schatz, Nanotechnology 17, 2813 (2006); E. M. Perassi, L. R. Canali, and E. A. Coronado, J. Phys. Chem. C 113, 6315 (2009); E. R. Encina, E. M. Perassi, and E. A. Coronado, J. Phys. Chem. A 113, 4489 (2009).

${ }^{4}$ S. A. Maier, P. G. Kik, H. A. Atwater, S. Meltzer, E. Harel, B. E. Koel, and A. A. G. Requicha, Nature Mater. 2, 229 (2003); M. I. Stockman, S. V. Faleev, and D. J. Bergman, Phys. Rev. Lett. 88, 067402 (2002); M. I. Stockman, ibid. 93, 137404 (2004).

${ }^{5}$ D. S. Citrin, Nano Lett. 4, 1561 (2004); A. Alù and N. Engheta, Phys. Rev. B 74, 205436 (2006).

${ }^{6}$ T. Baba, Nat. Photonics 2, 465 (2008).

${ }^{7}$ D. M. Newns, Phys. Rev. 178, 1123 (1969); E. Santos, M. T. M. Koper, and W. Schmickler, Chem. Phys. Lett. 419, 421 (2006).

${ }^{8}$ A. V. Malyshev, V. A. Malyshev, and J. Knoester, Nano Lett. 8, 2369 (2008); J. V. Hernández, L. D. Noordam, and F. Robicheaux, J. Phys. Chem. B 109, 15808 (2005); T. D. Backes and D. S. Citrin, Phys. Rev. B 78, 153407 (2008).

${ }^{9}$ V. A. Markel and A. K. Sarychev, Phys. Rev. B 75, 085426 (2007).

${ }^{10}$ S. Y. Park and D. Stroud, Phys. Rev. B 69, 125418 (2004).

${ }^{11}$ E. N. Economou, Green's Functions in Quantum Physics, 3rd ed.
(Springer, Heidelberg, 2006).

${ }^{12}$ H. M. Pastawski and E. Medina, Rev. Mex. Fis. S 47(1), 1 (2001); see arXiv:cond-mat/0103219, and references therein.

${ }^{13}$ D. J. Thouless, J. Phys. C 5, 77 (1972); H. M. Pastawski, J. F. Weisz, and S. Albornoz, Phys. Rev. B 28, 6896 (1983); E. Kilic, Appl. Math. Comput. 197, 345 (2008).

${ }^{14}$ A. D. Dente, R. A. Bustos-Marún, and H. M. Pastawski, Phys. Rev. A 78, 062116 (2008).

${ }^{15}$ H. Hogreve, Phys. Lett. A 201, 111 (1995); P. Serra, S. Kais, and N. Moiseyev, Phys. Rev. A 64, 062502 (2001); A. M. Pupasov, B. F. Samsonov, and J.-M. Sparenberg, ibid. 77, 012724 (2008).

${ }^{16}$ I. Rotter, J. Phys. A: Math. Theor. 42, 153001 (2009), and references therein.

${ }^{17}$ G. A. Álvarez, E. P. Danieli, P. R. Levstein, and H. M. Pastawski, J. Chem. Phys. 124, 194507 (2006); E. P. Danieli, G. A Álvarez, P. R. Levstein, and H. M. Pastawski, Solid State Commun. 141, 422 (2007).

${ }^{18}$ P. Nordlander, C. Oubre, E. Prodan, K. Li, and M. I. Stockman, Nano Lett. 4, 899 (2004).

${ }^{19}$ G. L. Liu, Y. Yin, S. Kunchakarra, B. Mukherjee, D. Gerion, S. D. Jett, D. G. Bear, J. W. Gray, A. P. Alivisatos, L. P. Lee, and F. F. Chen, Nat. Nanotechnol. 1, 47 (2006).

${ }^{20}$ L. A. Sweatlock, S. A. Maier, and H. A. Atwater, Proceedings of the 53rd Electronic Components and Technology Conference, New Orleans, 2003 (IEEE, Washington DC, 2003), p. 1648; H. L. Calvo, E. P. Danieli, and H. M. Pastawski, J. Phys. B 398, 317 (2007); L. Gutiérrez, A. Díaz-de-Anda, J. Flores, R. A. Méndez-Sánchez, G. Monsivais, and A. Morales, Phys. Rev. Lett. 97, 114301 (2006). 